\title{
Flows in the solar atmosphere due to the eruptions on the 15th July, 2002^
}

\author{
L. K. Harra ${ }^{1}$, P. Démoulin ${ }^{2}$, C. H. Mandrini ${ }^{3}$, S. A. Matthews ${ }^{1}$, L. van Driel-Gesztelyi ${ }^{1,2,4}$, \\ J. L. Culhane ${ }^{1}$, and L. Fletcher ${ }^{5}$
}

${ }^{1}$ Mullard Space Science Laboratory, University College London, Holmbury St.Mary, Dorking, Surrey, RH5 6NT, UK e-mail: lkh@mssl.ucl.ac.uk

2 Observatoire de Paris, section Meudon, LESIA (CNRS), 92195 Meudon Principal Cedex, France e-mail: Pascal.Demoulin@obspm. fr

${ }^{3}$ Instituto de Astronomía y Física del Espacio, CONICET-UBA, CC. 67 Suc. 28, 1428 Buenos Aires, Argentina e-mail: mandrini@iafe.uba.ar

${ }^{4}$ Konkoly Observatory of the Hungarian Academy of Sciences, PO Box 67, 1525 Budapest, Hungary e-mail: lvdg@mssl.ucl.ac.uk

5 Department of Physics and Astronomy, University of Glasgow, Glasgow G12 8QQ, UK e-mail: 1.fletcher@physics.gla.ac.uk

Received 2 March 2005 / Accepted 14 April 2005

\begin{abstract}
Which kind of flows are present during flares? Are they compatible with the present understanding of energy release and which model best describes the observations? We analyze successive flare events in order to answer these questions. The flares were observed in the magnetically complex NOAA active region (AR) 10030 on 15 July 2002. One of them is of GOES $\mathrm{X}$-class. The description of these flares and how they relate to the break-out model is presented in Gary \& Moore (2004). The Coronal Diagnostic Spectrometer on board SOHO observed this active region for around $14 \mathrm{~h}$. The observed emission lines provided data from the transition region to the corona with a field of view covering more than half of the active region. In this paper we analyse the spatially resolved flows seen in the atmosphere from the preflare to the flare stages. We find evidence for evaporation occurring before the impulsive phase. During the main phase, the ongoing magnetic reconnection is demonstrated by upflows located at the edges of the flare loops (while downflows are found in the flare loops themselves). We also report the impact of a filament eruption on the atmosphere, with flows up to $300 \mathrm{~km} \mathrm{~s}^{-1}$ observed at transition-region temperatures in regions well away from the location of the pre-eruptive filament. Our results are consistent with the predictions of the break out model before the impulsive phase of the flare; while, as the flare progresses, the directions of the flows are consistent with flare models invoking evaporation followed by cooling and downward plasma motions in the flare loops.
\end{abstract}

Key words. Sun: flares - Sun: UV radiation - Sun: corona - Sun: coronal mass ejections (CMEs)

\section{Introduction}

Understanding and predicting the flaring process on the Sun remains a focus in solar physics. Much progress has been made in both the theory and observational sides of flare physics, but one of the areas with limited information is that of observing the related flows spectroscopically.

Bulk plasma flows in flares, arising from the rapid heating of the lower solar atmosphere, provide a means of diagnosing the energy input to the lower atmosphere by beams or a conduction front, and may therefore help understand flare energy release models (generally accepted as involving magnetic reconnection, e.g. Kopp \& Pneuman 1976; Cargill \& Priest 1983). Single-fluid hydrodynamic models of chromospheric

* Figures 4-7 and 9 are only available in electronic form at http://www. edpsciences.org evaporation (e.g. Fisher et al. 1985a,b; Mariska et al. 1989) predict two distinct modes of evaporation: the "explosive" evaporation associated with rapid beam energy input - such that the radiative and conductive timescales are longer than the heating timescale - and "gentle" evaporation, when the reverse is true. The strong upflows of hot plasma (a few times $10^{6} \mathrm{~K}$ to $10^{7} \mathrm{~K}$ at a few hundred $\mathrm{km} \mathrm{s}^{-1}$ ) predicted from explosive models are rarely observed, although the Yohkoh-Bragg Crystal Spectrometer (BCS) had some success in measuring strong blueshifts, in full Sun spectra, in the early impulsive phase (see Culhane et al. 1994; Wuelser et al. 1994; Silva et al. 1997). The problems with detecting these strong flows may be in part observational: the flows are high-temperature and highvelocity but have a relatively low emission measure. From a theoretical point of view, recent work by Warren \& Doschek (2005) presents a model that can explain the discrepancies by 
computing multiple loop time dependent hydrodynamic simulations of flares. Their results are consistent with full Sun flare spectra from BCS.

Spatially resolved spectroscopic observations in the corona and transition region have been sparse. Teriaca et al. (2003) used a combination of SOHO Coronal Diagnostic Spectrometer (CDS, Harrison et al. 1995) observations with Sacramento Peak observations of the chromosphere. They obtained the first spatially resolved observations of the velocity field during the impulsive phase of a flare. They found that strong downflows (up to $40 \mathrm{~km} \mathrm{~s}^{-1}$ ) were measured in the chromosphere and strong upward motions were measured both in the transition region (up to $100 \mathrm{~km} \mathrm{~s}^{-1}$ ) and the corona (up to $160 \mathrm{~km} \mathrm{~s}^{-1}$ ). These signatures are consistent with chromospheric evaporation which is predicted by many theoretical models of flares. Similar results were found by Brosius (2003). He also noted that O III and O V emission in the transition region began to brighten a few minutes before the flare emission from Fe XIX appeared. However, spatially resolved spectroscopic observations of the preflare phase have yet to be obtained.

The post-flare phase has been analysed by Czaykowska et al. (1999). They used CDS data to search for evidence of chromospheric evaporation. They analysed Doppler velocity maps in emission lines of $\mathrm{O}$ V, Fe XVI and Fe XIX (with temperatures of formation $0.25,2.5$ and $8 \mathrm{MK}$ respectively). Bright downflowing plasma seemed to be dominant in the regions that coincided with the ends of the flare loops seen in the Extreme-Ultraviolet Imaging Telescope (EIT, Delaboudiniére et al. 1995). Strong upflowing plasma was seen in the weaker regions outside these bright footpoints. This observation is particularly significant in that the characteristic pattern of redand blue-shifts provides circumstantial evidence of a coronal magnetic reconnection process ongoing after the flare impulsive phase. The largest velocities measured were in the Fe XIX emission line. There is a significant advantage in having spatial resolution - however the drawback (as in our study) is that it took many minutes to build-up each image in this case.

Observations have been made using CDS without rastering an image i.e. the slit position is fixed. This allows good temporal resolution but with a loss of spatial information. Brosius and Phillips (2004) studied light curves and flows during a large flare. The slit was located in the region where the flare originated. They found evidence of low level evaporation in precursor events. This was seen in He II with upflows of $40 \mathrm{~km} \mathrm{~s}^{-1}$ observed. The Fe XIX coronal emission started to rise after the second precursor event. During the impulsive phase, there are downflows seen in the cooler O III, O IV, O V and He II emission lines which provide momentum balance to the upflowing coronal material. The velocities are of the order of tens of $\mathrm{km} \mathrm{s}^{-1}$.

Recent work carried out by Gary \& Moore (2004) described the eruption of a helical magnetic flux tube in a large X-class flare that took place on the 15 July 2002. They used multiinstrument observations including the Transition Region and Coronal Explorer (TRACE, Handy et al. 1999) high resolution $1600 \AA$ images and vector magnetograms from the Marshall Space Flight Center (MSFC). The magnetic field of the active region is mainly a quadrupolar field with a strong magnetic shear. Gary \& Moore proposed that the core helical field erupted after the initiation of the quadrupolar flare, hence supporting the breakout model of Antiochos et al. (1999) instead of the standard flare model that requires the eruption to trigger the whole process of reconnection. They found initial brightenings in TRACE $1600 \AA$ images that are consistent with reconnection at the magnetic null point located above the erupting core field. This was confirmed by Li et al. (2005). They found that the ribbons, associated to the quadrupolar reconnection, are not only present, but are also shifted in location (with a nearly constant velocity) well before the impulsive phase of the flare (at least $5 \mathrm{~min}$ ). During this time period they also found that the filament present in the core field is accelerated upward. Both the ribbon motion and the filament acceleration suddenly increased when the impulsive phase started. The $\mathrm{H} \alpha$ filament eruption is then followed by an erupting helix-like structure observed by TRACE. The above two papers demonstrate that the quadrupolar reconnection has a key role in the eruption: it started before, and the reconnection rate is enhanced, during the impulsive phase. At the beginning of our research we analyzed extensively the TRACE and Michelson Doppler Imager (MDI, Scherrer et al. 1995) data and we independently reached the same conclusion as Gary \& Moore, and Li et al. on the role of the quadrupolar reconnection.

In this paper we analyze in detail the flows observed in the 15 July event, from preflare to flare, making use of spectroscopic data sets from the SOHO-CDS. This allows us to observe spatially resolved velocities in the transition region and corona during the preflare and impulsive flare stages. We can then investigate whether there is any evidence of outflowing material before the impulsive phase of this complex event, as would be expected from evaporation in the breakout model. This event allows us to search for that due to the long time coverage as well as good spatial coverage of the spectroscopic observations.

\section{Instruments and data}

The MDI instrument on board SOHO images the Sun on a $1024 \times 1024$ CCD camera through a series of increasingly narrow filters. The final elements, a pair of tunable Michelson interferometers, enable MDI to record filtergrams with a FWHM bandwidth of $100 \mathrm{~m} \AA$. In this paper, we analyse 5 min averaged magnetograms of the full disk with a 96 min cadence and a pixel size of $1.98^{\prime \prime}$.

SOHO-CDS consists of the Normal Incidence Spectrometer (NIS) and the Grazing Incidence Spectrometer (GIS) covering the wavelength range 150-800 $\AA$. This range has spectral lines formed in the temperature range $T_{\mathrm{e}}=10^{4}-10^{6} \mathrm{~K}$. The CDS observation was made using the FLARE_AR study. The exposure time at each slit position was $8 \mathrm{~s}$, and the slit was rastered over 45 locations covering $183^{\prime \prime} \times 183^{\prime \prime}$ field of view. Five spectral windows were used

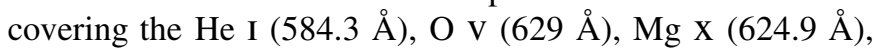
Fe XVI (360.8 $\AA$ ) and Fe XIX (592.2 $\AA$ ) ion spectra. We concentrated on analysing the cleanest optically thin emission lines, although it should be noted that He I showed similar 


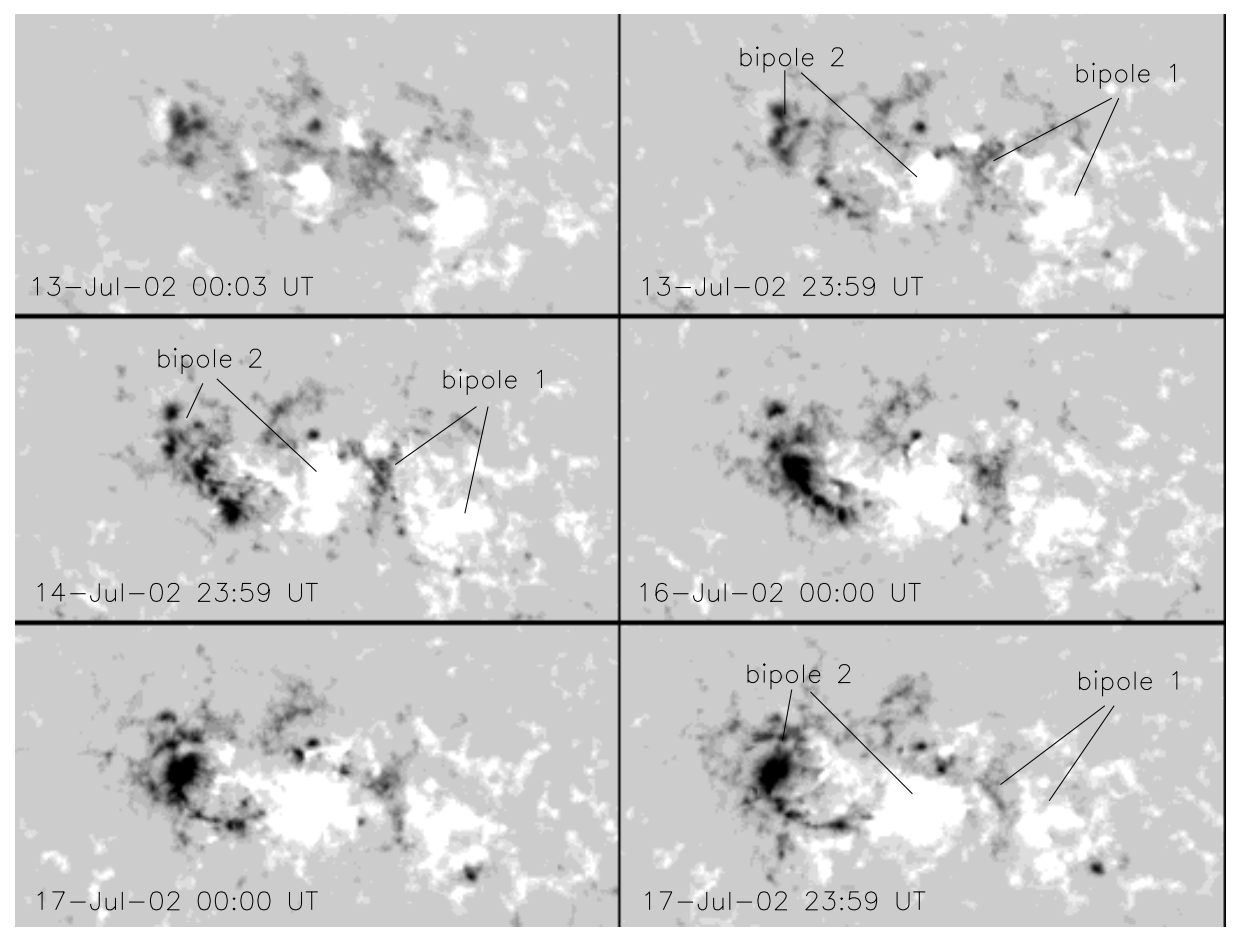

Fig. 1. De-rotated co-aligned portions of MDI full-disk images (2"/pixel) showing NOAA AR 30 between 13-17 July 2002 . The polarities of the two main bipoles are indicated. Due to the usual divergence of magnetic polarities during flux emergence the following (negative polarity) part of bipole 1 is being "invaded" and gradually removed, presumably via magnetic cancellation, by the leading (positive polarity) magnetic fields of bipole 2. We propose that this interaction between the two main bipoles was the cause of the quadrupolar confined flare (phase A of the complex flare, see text). White: positive, black: negative polarity.

Doppler shifts to the $\mathrm{O} \mathrm{v}$ emission line. These spectral lines measure plasma from the chromosphere through the transition region and into the corona, with one high temperature emission line that appears bright only during flares. The first raster started at 07:54 UT, and the last raster started at 22:29 UT on 15 July 2002 . Each raster takes $\approx 11 \mathrm{~min}$ to complete. The raster starts on the right-hand side of the "image".

For each spatial pixel we fitted the spectral lines with a Gaussian profile. We carried out fitting on all spatial pixels that have an intensity greater than $10 \%$ of the maximum intensity for $\mathrm{O} \mathrm{V}$ and Fe XVI. For the weaker Fe XIX emission line we fitted all pixels with an intensity greater than $40 \%$ of the maximum intensity in order to ensure good signal to noise relation in the line profiles. We made velocity maps from the spectral information in the images by measuring the centroid shifts of the Gaussian profiles fitted to each pixel. The "rest" wavelength was assumed to be that of the spectrum of all the pixels averaged over the entire raster scan during the preflare phase so no large Doppler shifts could bias the rest wavelength. We have hence obtained a "relative" velocity. The errors on the spectral fitting are small $\left(<5 \mathrm{~km} \mathrm{~s}^{-1}\right)$ due to the high count rate in the emission lines chosen.

TRACE observed the pre-flare stage and the flare itself on the 15 July. Gary \& Moore (2004) describe the flare stage in detail. TRACE was observing in flare mode with the $1600 \AA$ filter. The pixel size is $0.5^{\prime \prime}$ giving a spatial resolution of $1.0^{\prime \prime}$. We co-aligned the TRACE data by overlaying the sunspots visible in the TRACE $1600 \AA$ channel and the MDI high resolution continuum images.
The EUV imaging observations were obtained by EIT that observes the full Sun regularly with four different filters and with a pixel size of $2.6^{\prime \prime}$. We use the observations made with the $195 \AA$ filter. The co-alignment between CDS and EIT was checked for accuracy by cross-correlating the He I in CDS and He II $304 \AA$ in EIT.

\section{Evolution of the active region}

The active region was built up by several subsequent episodes of flux emergence, but can be regarded as quadrupolar, consisting of two main bipoles arranged roughly in E-W direction (Fig. 1). The younger bipole 2 started emerging on 10 July trailing the pre-existing bipole 1. Its leading (positive polarity) spots were approaching the fragmented following (negative polarity) region of bipole 1 , while moving westward. The evolution of bipole 2 involved a second episode of flux emergence: on 13 July a new bipole appeared in it. The new bipole expanded and its magnetic flux grew quickly. By 15 July bipole 2 became "double-headed", having two major leading spots as a result of the two flux emergence episodes.

After 15 July systematic motions continued, leading to the gradual disappearance of the negative polarity of bipole 1 presumably via magnetic cancellation (Fig. 1). There were two major filaments present in the active region: one along the inversion line of bipole 1 , another along the inversion line between bipoles 1 and 2 . 


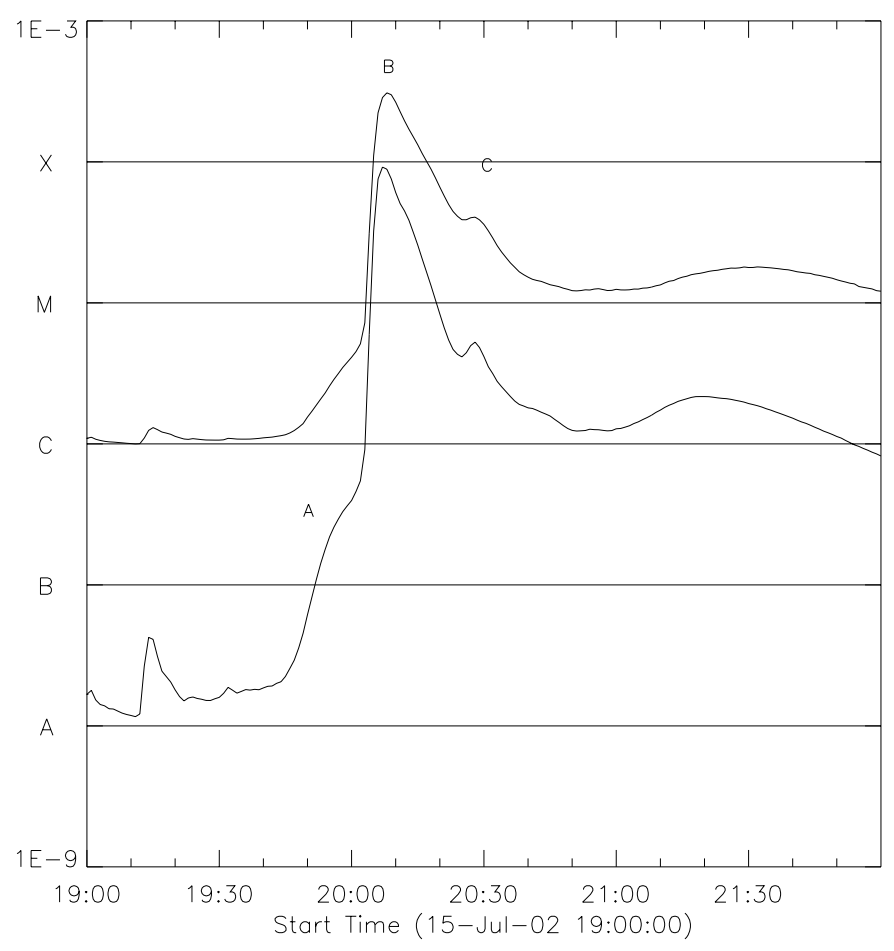

Fig. 2. The GOES light curve for the 15 July flare. There are 3 main stages for the flare labeled A, B and C. There are two filament eruptions - the first occurs in phase $\mathrm{B}$ and the second in phase $\mathrm{C}$. The bottom curve shows the $(0.5-4 \AA)$ energy range and the top curve shows the (1-8 $\AA$ ) energy range.

\section{The different phases of the flare}

The complex flare we analyse here had three stages (Fig. 2). Phase A was a quadrupolar confined flare with footpoints in all the four polarities of bipoles 1 and 2 (start $\approx 19: 45$ UT). Phase $\mathrm{B}$ had a filament eruption followed by a long duration flare (start $\approx 20: 00$ UT) between the two bipoles. Finally, phase $\mathrm{C}$ had a filament eruption and a long duration event (LDE) occurred in bipole 1 (start $\approx 20: 25 \mathrm{UT}$ ). The TRACE movie for these events can be viewed online in Gary \& Moore (http://science.nasa.gov/ssl/PAD/SOLAR/papers/ gary/HelixPaper.html).

\subsection{Phase A: quadrupolar reconnection}

The CDS field of view concentrated on bipole 2 and observed only the eastern edge of bipole 1 . The CDS observations included the intense, clean $629 \AA \mathrm{O} \mathrm{V}$ transition region line with a formation temperature of $\approx 0.25 \mathrm{MK}$. Not surprisingly the location of the TRACE $1600 \AA$ emission is approximately the same as the transition region emission seen by CDS (Fig. 3). In addition the coronal Fe XVI intensity is shown, with a formation temperature of 2.5 MK. The Fe XVI emission shows the location of the coronal loops which are present only inside bipoles 1 and 2 .

Gary \& Moore (2004) analyzed the preflare X-ray emission increase which starts at 19:45 UT and the TRACE $1600 \AA$ emission increase that starts at 19:50 UT. They concluded that there were small brightenings at the separatrix footpoints which indicates reconnection at the magnetic null of the quadrupolar configuration (bipoles 1 and 2). However, they had no direct evidence for reconnection high in the corona.

With CDS data we searched for evidence of evaporation during the beginning of the flare before the dramatic filament eruptions. The first $\mathrm{O} \mathrm{V}$ velocity map in which we observe strong blue-shifts is in the raster starting at 19:36 UT (Fig. 3). These velocities reach absolute values of $\approx 40 \mathrm{~km} \mathrm{~s}^{-1}$. A stronger emission intensity is present also in the flow region. This occurs right at the beginning of phase A $(\approx 19: 43$ UT) as estimated from the scanning of the CDS instrument. We interpret this brightening and the corresponding flows as evidence of chromospheric evaporation which indicates that magnetic reconnection has taken place. This is likely to have been induced by the moving magnetic features around the northern leading spot of bipole 2 .

The observations in Phase A, between 19:47 and 19:59 UT, show some strong blue-shifts in the $\mathrm{O} \mathrm{V}$ emission line in two locations (at $\approx 110225$ and -15230 in Fig. 4). During these times GOES data show a slow rise to mid-C flare level (Fig. 2). The western region of strong blue-shifted emission in the $\mathrm{O} v$ lies at the end of the bright Fe XVI emission, strongly suggestive of a footpoint of a coronal loop showing evaporation (Fig. 4). The same mechanism also provides the most likely explanation of the eastern region of strong blue-shifted emission. The bright emission seen in EIT and Fe XVI correspond to dense and heated loops in bipole 2 that we interpret as the result of the quadrupolar reconnection (Gary \& Moore 2004).

\subsection{Phase B: the first filament eruption and associated flare}

In phase B there is an impulsive rise seen in the GOES light curve at $\approx 20: 02$ UT (Fig. 2). The filament eruption located between bipole 1 and bipole 2 was observed with a high cadence by TRACE. The observations were interpreted as a central breakout eruption by Gary \& Moore (2004). Unfortunately due to the rastering required by CDS in order to build up an image, CDS missed the main filament eruption which lasted only a couple of minutes. CDS observations did however observe the response in the atmosphere after the filament lift-off.

The flows observed in both the transition region and corona have increased in magnitude during this period. The first CDS raster during this period is shown in Fig. 5. Evidence for the eruption is seen with strong outflows in the Fe XVI (negative velocities reaching $\approx 50 \mathrm{~km} \mathrm{~s}^{-1}$ in the region around $(-70$, 320) arcsecs). These velocities are seen around 20:05 UT (taking into account the CDS scanning). In the next CDS raster, at 20:10 UT (Fig. 6), there is also a strong upflow seen in the Fe XVI, but also in the $\mathrm{O} V$ (centred at $\approx(-10,340)$ arcsecs) at around 20:12 UT. In both lines the velocities reached an absolute value of $150 \mathrm{~km} \mathrm{~s}^{-1}$. The region of blue shift is much more extended and shifted toward the north than the initial location of the filament (around 0,250 arcsecs). The shift is consistent with the apparent motion of the eruptive filament observed with TRACE and in $\mathrm{H} \alpha$. However, the velocities observed by CDS are much lower than the $\mathrm{H} \alpha$ velocity of the 

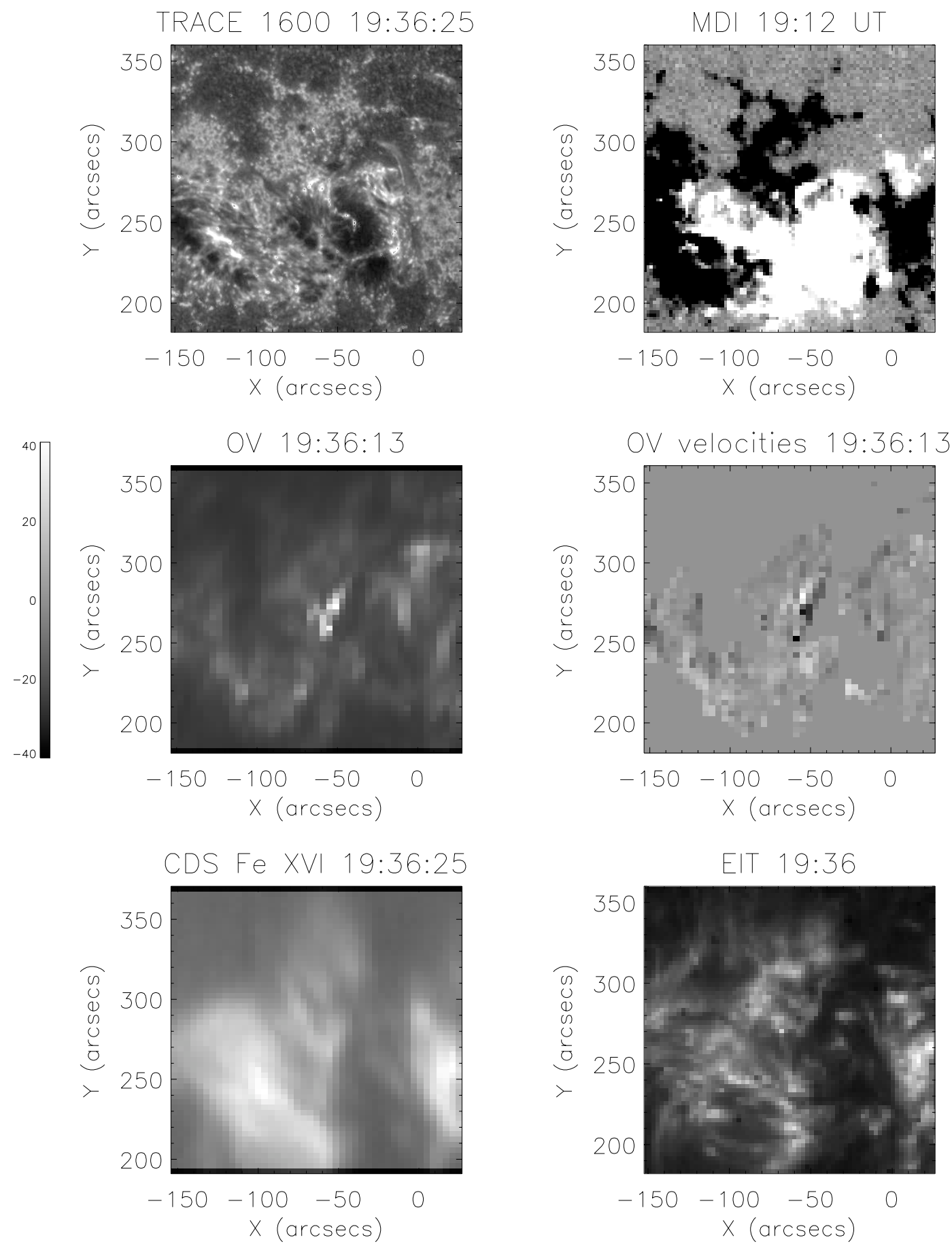

Fig. 3. Beginning of phase A (quadrupolar reconnection). In this and in all figures, the times shown on the CDS images correspond to the start time of the raster (from right to left with a duration $\approx 11 \mathrm{~min}$ ). The top left panel shows the TRACE $1600 \AA$ image just before there is an increase in coronal emission. The top right panel shows the longitudinal magnetic field within the CDS field of view. The middle row shows the $\mathrm{O} \mathrm{v}$ intensity (left) and Doppler velocity (right) image. The brightening in O v occurs at $\approx 19: 43 \mathrm{UT}$ at the start of Phase A (taking into account the scanning delay occurring from right to left). The bottom left panel shows the Fe XVI emission (mostly located inside bipoles 1 and 2). The bottom right panel shows the EIT intensity $195 \AA$ A image.

filament $\left(\approx-400 \mathrm{~km} \mathrm{~s}^{-1}\right.$ at $\approx 20: 03: 40 \mathrm{UT}$, Li et al. 2005). We conclude that CDS did not observe the erupting filament, but rather the surrounding coronal plasma or/and part of the filament plasma when it was already heated to transitionregion/coronal temperatures. We note that the slow scanning of CDS, from right to left, needs to be taken into account to understand the observations shown in Figs. 5 and 6 as follows. At 19:59 UT, the filament eruption has not yet started, and CDS observed the eruption only later on giving the false impression that the strong velocities are only in the middle part of the raster (Fig. 5). At 20:03 UT, when the strong acceleration of the filament started (Fig. 6 of Li et al. 2005), CDS 
scanning had already gone beyond the filament location, so CDS could only observe the eastern side effects of the eruption. The next raster detected the eruption from the beginning of the scan, but the filament material has already moved out of the field of view (Fig. 6). All together, the two rasters give a better idea of the true extension of the eruption: while CDS scanning missed the filament eruption itself, it shows that the eruption affects a much larger area than detected in $\mathrm{H} \alpha$. Indeed, this is an evidence that, at least, a large part of the stressed magnetic core erupted, and that the $\mathrm{H} \alpha$ filament shows only a small fraction of it.

By the time of the CDS raster shown in Fig. 6 flare loops have formed: an arcade of hot flare loops connects the negative polarity of bipole 1 and the positive polarity of bipole 2 . The largest blue-shifts seen in Fe XVI are cospatial with the weaker emission at the edge of the brightest loop system. This is direct evidence of "evaporation" taking place as a consequence of energy released in the corona. Red shifts are also seen in between the two "ribbons" of blue shifts in Fig. 6 - with the largest downflows close to the brightest region of Fe XVI emission. This indicates that cooling had begun in these coronal loops (which were reconnected before the ones linking the two "ribbons" of blue shifts).

The flare continues with strong blue-shifts seen in Fe XVI, and Fe XIX emission becoming strong (Fig. 7). Both the Fe XVI and Fe XIX emissions show the strongest blue shifts towards the edges of these loops. The location of the blue shifts is consistent with the flare ribbons seen in TRACE. There are also downflows in between, i.e. around the loop tops. It is difficult to tell with the limited resolution if the downflows are at the loop top or exist in the loop legs. The latter may be expected from the traditional model of magnetic reconnection with material that is cooling and draining down the loop legs. The two blue shifted "ribbons" separate with time and bright loops are seen at this stage in EIT. Within the limit of CDS temporal resolution (which affects the spatial extension of the observed emissions), the EIT loops are co-spatial with Fe XVI, and Fe XIX emission (in particular EIT loops are in between the two blue shifted "ribbons"). The main difference is that Fe emissions are more intense around the center of the loop system. We interpret both EIT and CDS emissions as evidence of flare loops. All these features are a consequence of magnetic reconnection occurring at larger heights under the erupting filament.

We combine the information given by CDS (line of sight velocities, $v_{l}$ ) with magnetic field extrapolations to determine the direction in which the plasma flows along observed loops, as done by Harra et al. (2004). To do so we assume that the velocities measured in CDS are mainly due to plasma flows along field lines, and we use the approximation of a static magnetic field. Under these assumptions, $\boldsymbol{v}$ is parallel to $\boldsymbol{B}$, and we have $\boldsymbol{v}=\frac{v_{l}}{B_{l}} \boldsymbol{B}$ (Eq. (1) in Harra et al. 2004). Then, with $v_{l}$ from CDS data and $\boldsymbol{B}$ and $B_{l}$ from a magnetic field model, it is possible to find the plasma velocity along these loops. We use CDS and TRACE observations at 20:22 UT, where we can see the flare ribbons and the connecting loops. Taking the MDI magnetogram closest in time, we compute the coronal magnetic field under the linear (or constant $\alpha$ ) force-free field assumption, $\boldsymbol{\nabla} \times \boldsymbol{B}=\alpha \boldsymbol{B}$, using a fast Fourier transform method, as proposed by Alissandrakis et al. (1981). The value of the free parameter $\alpha$ is determined to give the best global fit to TRACE flare loops at 20:22 UT. We find that $B_{l}$ points towards the observer along the eastern portion of TRACE loops and progressively changes direction, pointing away from the observer, as we move westward along the field lines (Fig. 8). Combining this with CDS Fe XVI velocity maps in Fig. 7 and the direction of $\boldsymbol{B}$ (shown by the arrows in Fig. 8), we conclude that the plasma flows upwards at the outer edges of the Fe XVI emission and downwards in all the red-shifted portion. This is an indication of on going evaporation in the newly formed flare loops and of cooling of the inner loops.

\subsection{Phase C: second filament eruption and associated flare}

There is a second filament eruption seen in TRACE at $\approx 20: 25$ UT which occurs in bipole 1 mainly outside the CDS field of view that we interpret as a lateral breakout eruption (as in the flare analyzed by Aulanier et al. (2000), while it is worth noticing that the magnetic configuration and its evolution are different). There is no evidence of flows seen in the coronal lines in the region of the filament eruption since the intensity is very low. However, there are dramatic blue-shifts seen over a large area in $\mathrm{O} v$ (North of the central right image in Fig. 9). This shows approximately the extent of the disruption that the filament eruption caused in one CDS scan (since the relative timing of the scan and of the eruption was much more favorable in this case than in the first eruption). The flows reach velocities with absolute values of nearly $300 \mathrm{~km} \mathrm{~s}^{-1}$. This flow magnitude is comparable to the $\mathrm{H} \alpha$ velocities found by $\mathrm{Li}$ et al. (2005) for the first filament eruption, showing that such flow magnitudes can be detected by CDS. It points, once more, to the importance of having a high temporal cadence to detect these high speeds in such eruptions.

We also notice that there is still ongoing blue-shifted emission at the edge of the loop system of the first eruption (together with downflows seen in the loop tops). This shows that the second filament eruption occurred while there was still energy being released in the reconnection process of the first eruption.

\section{Conclusion}

We describe the flows observed in the transition region and corona seen by SOHO-CDS during a complex X-class flare. The flare occurred in three main stages, including two filament eruptions.

The first stage of the flare (phase A) was characterized by a gradual rise in soft X-ray flux. There was evidence for upflowing material from the lower atmosphere (transition region). This was also seen, in another flare, by Brosius \& Phillips (2004), whose single slit observations had the time cadence to observe the precursors before the impulsive phase but lacked spatial resolution. In the present paper we find evidence for evaporation at this early stage through upflows seen in spatially resolved $\mathrm{O} \mathrm{V}$ spectra and in brightenings observed with TRACE. The presence of evaporation lends support to 

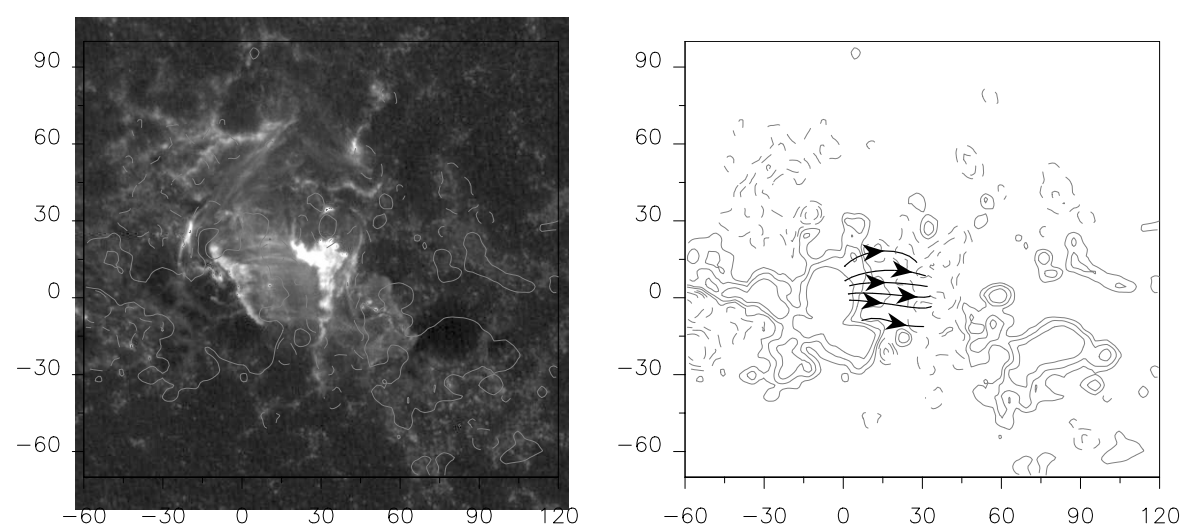

Fig. 8. The left figure shows the TRACE intensity $1600 \AA$ Amage at 20:22 UT. Flare loops are observed connecting the flare ribbons in phase B. The right figure corresponds to a magnetic field model showing a set of field lines matching the TRACE loops. The arrow shows the direction of $\boldsymbol{B}$ and the axes are in Mm. Three isocontours of the line of sight magnetic field are included $( \pm 100,250,500 \mathrm{G})$ in this figure, while only one is shown in the left figure $( \pm 100 \mathrm{G})$. The positive (negative) values are shown with continuous (dashed) lines.

the idea that magnetic reconnection has already started to release energy. This evidence of reconnection in a large-scale quadrupolar configuration preceding the first filament eruption is supportive of the breakout model (see Antiochos et al. 1999).

The first impulsive stage (phase B) was associated to the first filament eruption in the central part of the quadrupolar configuration. Flare loops were formed in the coronal lines of Fe XVI (2.5 MK) and Fe XIX (8 MK). The loops showed blueshifts at the weaker emission regions at the edge of the loop system and red shifts closer to the loop tops. The blue shifts reach a maximum value of $100 \mathrm{~km} \mathrm{~s}^{-1}$, but are generally around tens of $\mathrm{km} \mathrm{s}^{-1}$. These are consistent with the results of a statistical study of upflows carried out by Mariska (1994) who found that blue-shifts measured by the Yohkoh-BCS in the X-ray regime averaged between 50 and $80 \mathrm{~km} \mathrm{~s}^{-1}$.

By combining CDS line of sight velocities with magnetic field modelling, we calculate the direction of the flow of the plasma. We find that our results are consistent with upflows at the base of newly reconnected loops (the hottest ones: several MK), and with downflows in the flare loops observed by TRACE $(\approx 1 \mathrm{MK})$ during phase $\mathrm{B}$. The behaviour of the upflows and downflows are consistent with chromospheric evaporation in sequentially reconnected loops, with the upflowing plasma existing in newly formed loops that are not yet bright. This is also consistent with the observations of chromospheric evaporation described earlier by Czaykowska et al. (1999) and with the "standard" flare models described in the introduction.

The upflows in the transition region line $\mathrm{O} v$ are seen during the preflare stages before the plasma has been heated. However, there is no strong evidence of upflows in this line during the development of the flares. This is probably due to the increased dynamics of the plasma during flares: the plasma is heated quickly to coronal temperatures and the emission measure at transition temperature stays low.

The second filament eruption (phase C) occurred in the western lobe of the quadrupolar configuration in bipole 1 out of the CDS field of view. However we measured large Doppler shifts up to $300 \mathrm{~km} \mathrm{~s}^{-1}$ over a large area in the $\mathrm{O} \mathrm{V}$ line (around $80^{\prime \prime}$ broad). A similar large Doppler signal was also observed during the first filament eruption, but with a much less favourable timing of the eruption with respect to the CDS scan. CDS allows us to detect the plasma flows over a much more extended area than in $\mathrm{H} \alpha$ observations, where they only show the filament. This is evidence that the erupting magnetic configuration is much more extended than the erupting filament (in agreement with the large extension seen with EIT and LASCO in other events).

Understanding these flows before and during a flare is of the utmost importance for clarifying the flare process. Imaging spectroscopy is providing us with the ability to spatially resolve the flows. The Solar-B mission, which will be launched in 2006, is focused on understanding heating and flaring processes in active regions. The temporal resolution of the EUV Imaging Spectrometer (EIS) on Solar-B will be significantly better than that of SOHO-CDS for studying flares since it is purpose built for this, whereas SOHO-CDS was built to study the quiet Sun and coronal heating. Hence significant progress is expected, especially in the understanding of the flare trigger phase.

Acknowledgements. The authors thank the SOHO-MDI consortium for the MDI data and the Mullard Space Science Laboratory (MSSL) Solar UK Research Facility (SURF) for providing data for use in this publication. SOHO is a joint project by ESA and NASA. P.D. and C.H.M. acknowledge financial support from CNRS (France) and CONICET (Argentina) through their cooperative science program (05ARG0011). L.v.D.-G. was supported by the Hungarian government grant OTKA T038013. C.H.M. was supported by the Argentinean grants UBACyT X329 and PICT 12187 (ANPCyT). C.H.M. is grateful for an MSSL/PPARC visitor's grant. The authors acknowledge travel support from the Royal Society Joint Project (MSSL/UCL and Observatoire de Paris, Meudon). L.K.H. acknowledges PPARC for the award of an advanced fellowship. C.H.M. is a member of the Carrera del Investigador Científico (CONICET).

\section{References}

Alissandrakis, C. E. 1981, A\&A, 100, 197

Aulanier, G., DeLuca, E. E., Antiochos, S. K., McMullen, R. A., \& Golub, L. 2000, ApJ, 540, 1126

Antiochos, S., Devore, C. R., \& Klimchuk, J. A. 1999, ApJ, 510, 485

Brosius, J. W. 2003, ApJ, 586, 1417 
Brosius, J. W., \& Phillips, K. J. H. 2004, ApJ, 613, 580

Cargill, P., \& Priest, E. R. 1983, ApJ, 266, 383

Culhane, J. L., Phillips, A. T., Inda-Koide, M., et al. 1994, Sol. Phys., 153,307

Czaykowska, A., De Pontieu, B., Alexander, D., \& Rank, G. 1999, ApJ, 521, L75

Delaboudiniere, J.-P., Artzner, G. E., Brunaud, J., et al. 1995, Sol. Phys., 162, 291

Fisher, G. H., Canfield, R. C., \& McClymont, A. N. 1985a, ApJ, 289, 424

Fisher, G. H., Canfield, R. C., \& McClymont, A. N. 1985b, ApJ, 289, 414

Gary, G. A., \& Moore, R. L. 2004, ApJ, 611, 545

Handy, B. N., Acton, L. W., Kankelborg, C. C., et al. 1999, Sol. Phys., 187, 229

Harra, L. K., Mandrini, C. H., \& Matthews, S. A. 2004, Sol. Phys., 223,57

Harrison, R. A., Sawyer, E. C., Carter, M. K., et al. 1995, Sol. Phys., 162,233
Kopp, R. A., \& Pneuman, G. W. 1976, Sol. Phys., 50, 85

Li, J., Mickey, D. L., \& LaBonte, B. J. 2005, ApJ, 620, 1092

Liu, Y., \& Norton, A. 2001, MDI measurement errors: The magnetic perspective, SOI-technical note 01-44,

http://soi.stanford.edu/_data/_cal/mag_data.html \#nonlinearity

Mariska, J. T., Emslie, A., Gordon, \& Li, P. 1989, ApJ, 341, 1467

Mariska, J. T. 1994, ApJ, 434, 756

Silva, A. V. R., Wang, H., Gary, D. E., Nitta, N., \& Zirin, H. 1997, ApJ, 481, 978

Scherrer, P. H., Bogart, R. S., Bush, R. I., et al. 1995, Sol. Phys. 162, 129

Teriaca, L., Falchi, A., Cauzzi, G., Falciani, R., Smaldone, L. A., \& Andretta, V. 2003, ApJ, 588, 596

Warren, H. P., \& Doschek, G. A. 2005, ApJ, 618, L157

Wuelser, J.-P., Canfield, R. C., Acton, L. W., et al. 1994, ApJ, 424, 459 


\section{Online Material}


L. K. Harra et al.: Flows in the solar atmosphere, Online Material p 2
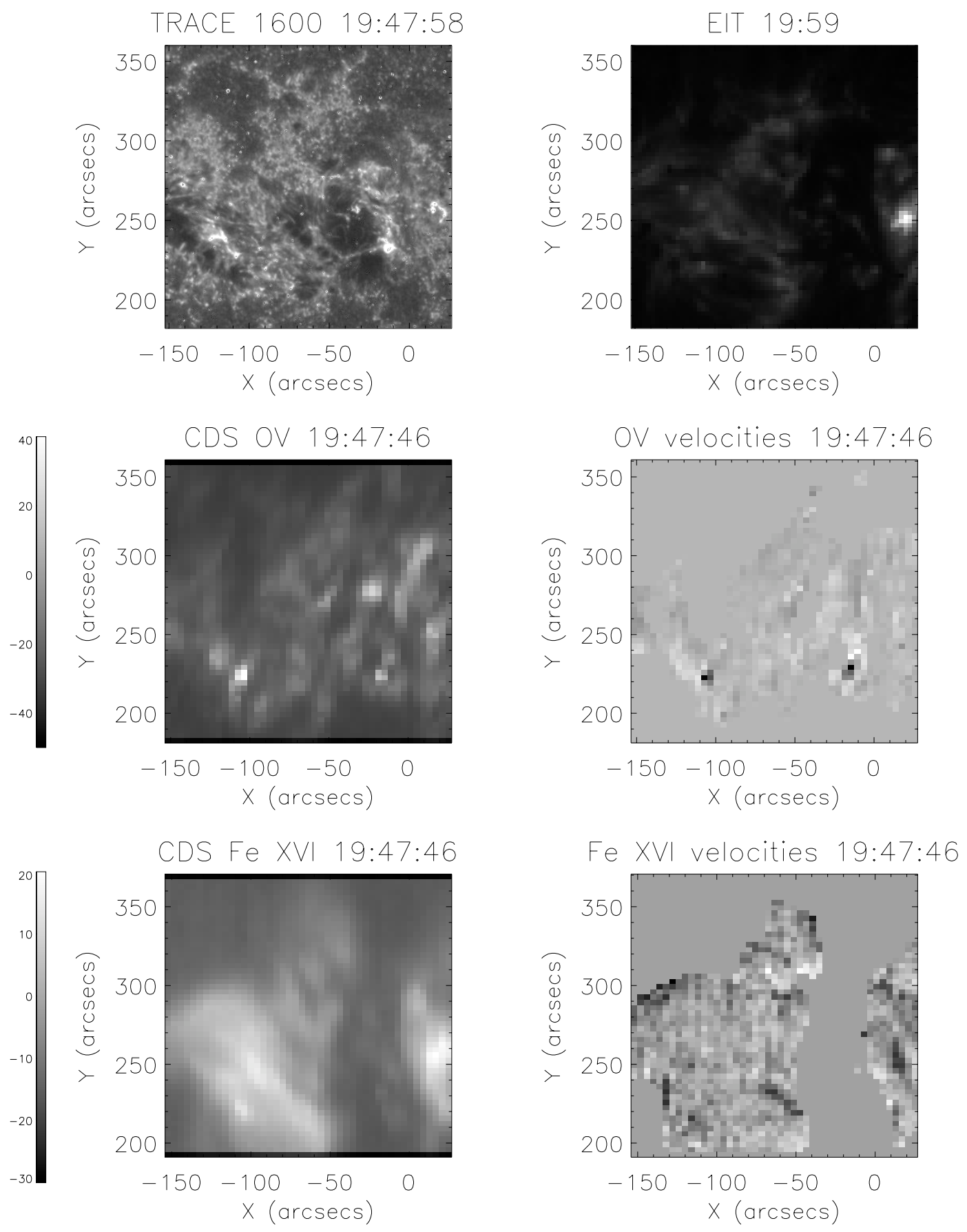

Fig. 4. Main phase A (quadrupolar reconnection). The top left panel shows the TRACE intensity $1600 \AA$ Amage over the CDS field of view (just before the impulsive phase of the flare). The top right panel shows the EIT $195 \AA$ A image. The middle row shows the O V intensity (left) and Doppler velocity (right) image. The bottom row shows the Fe XVI intensity (left) and Doppler velocity (right) image. 
L. K. Harra et al.: Flows in the solar atmosphere, Online Material p 3
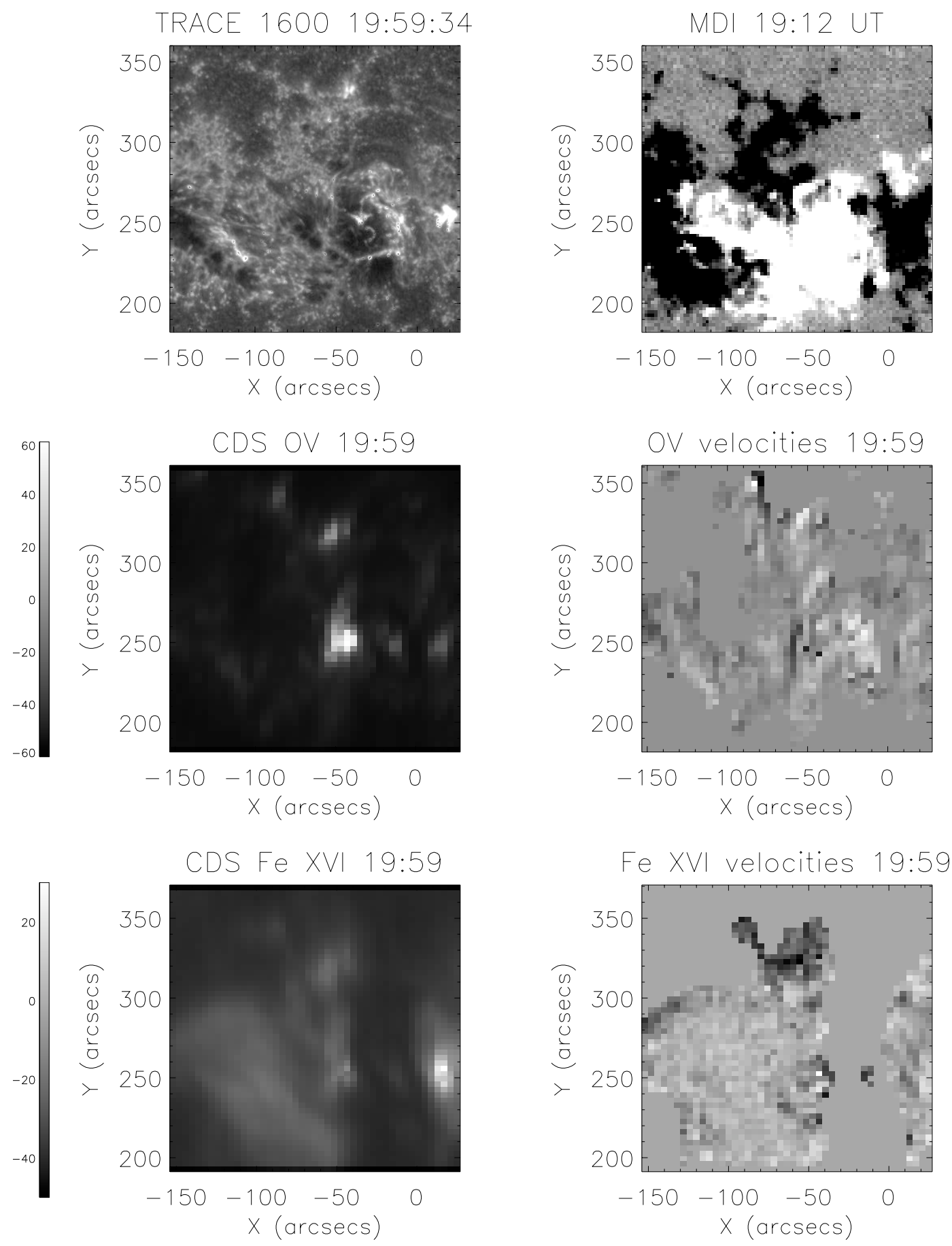

Fig. 5. Beginning of phase B (first filament eruption). The top left panel shows the TRACE image, and top right the MDI image. The middle row shows the $\mathrm{O} \mathrm{V}$ intensity image (left) and Doppler shift image (right). The main features are the brightenings associated to the quadrupolar reconnection (left row) and the negative (upward) velocities associated to the first filament eruption (better seen in the Fe XVI Doppler velocity image). At this stage the EIT image was saturated. The bottom row shows the Fe XVI intensity (left) and Doppler shift (right). 
L. K. Harra et al.: Flows in the solar atmosphere, Online Material p 4
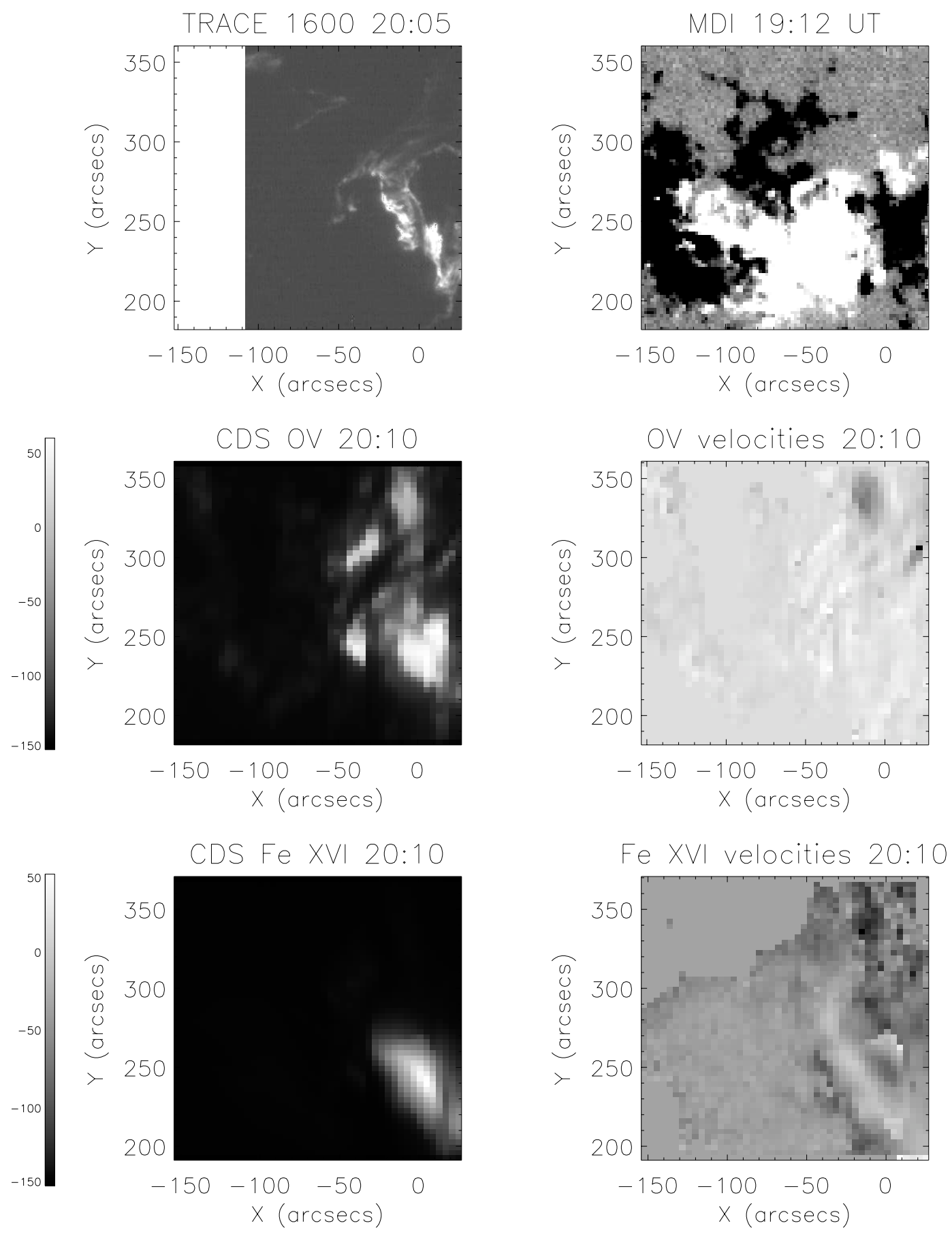

Fig. 6. Main phase B (first filament eruption). Same panels as in Fig. 5. The main flare features are the two ribbons observed with TRACE and with CDS O V and the flare loops observed with CDS Fe XVI. The main features of the filament eruption are the new O v brightenings (right and top half of the image) and the associated blue-shifted velocities (right panels). 
L. K. Harra et al.: Flows in the solar atmosphere, Online Material p 5
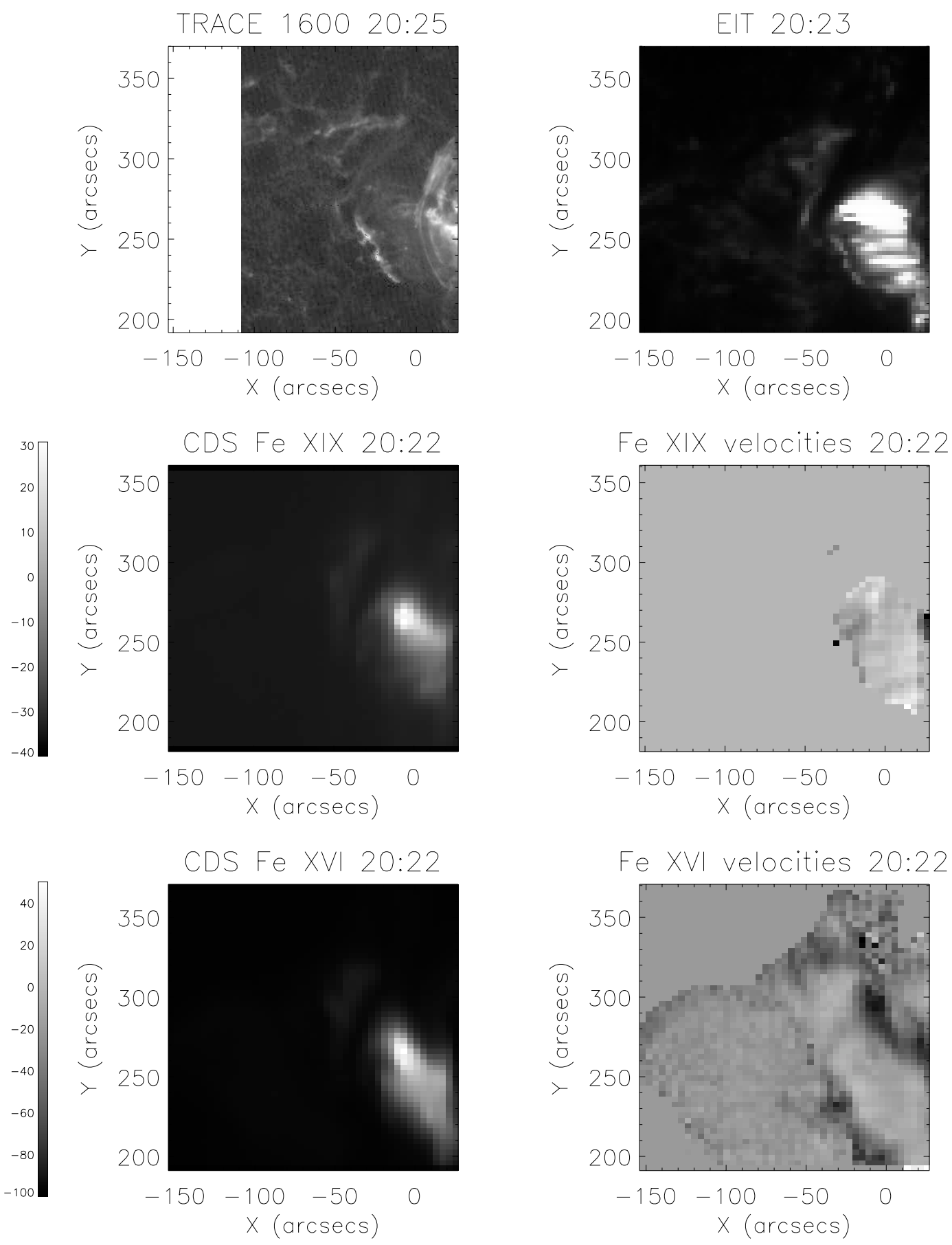

Fig. 7. End of phase B (first filament eruption). Same panels as in Fig. 4 except that the middle row shows the Fe XIX intensity image (left) and Doppler velocity (right). The main features are the flare loops and their associated characteristic flow pattern (blue on the borders, red in the center). 
L. K. Harra et al.: Flows in the solar atmosphere, Online Material $p 6$
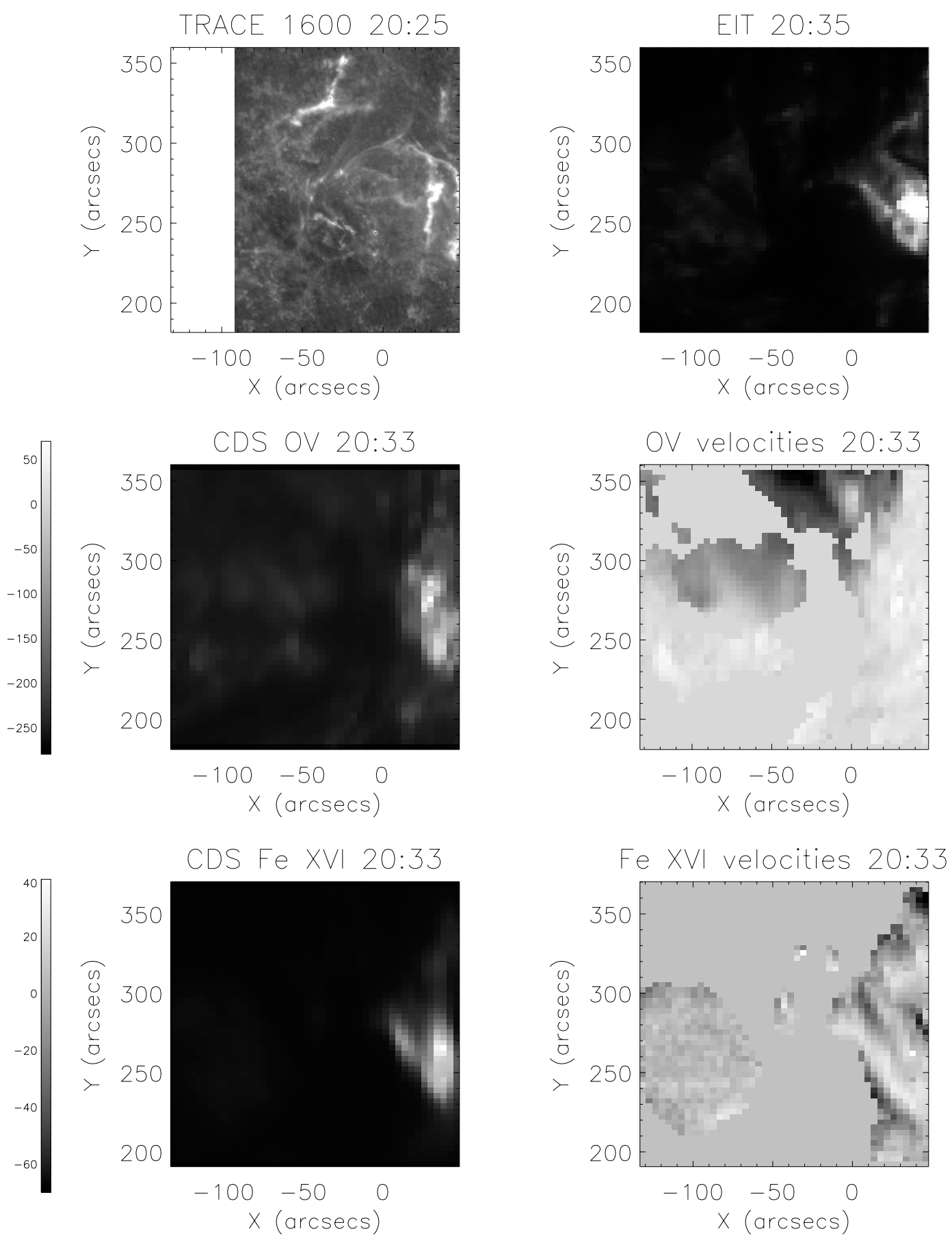

Fig. 9. Phase $\mathrm{C}$ (second filament eruption). Same panels as in Fig. 4. The main feature is the extended blue shifted region in $\mathrm{O} v$ tracing the outward filament ejection. 